\title{
Acaricidal Efficacy Against Ticks Delcid
}

\author{
Yuri V. Glazunov \\ ASRIVEA - Branch of Tyumen Scientific Centre SB RAS \\ Institutskay Str., Tyumen, Russia \\ E-mail: glazunovurii@mail.ru
}

\author{
Larisa Aleksandrovna Glazunova \\ candidate of veterinary sciences, associate professor, \\ FSBEI HE Northern Trans-Ural SAU \\ 7 Respubliky, Tyumen, Russia \\ E-mail: glazunovala@gausz.ru
}

\begin{abstract}
In the Northern Trans-Ural region ticks are dangerous to animals as carriers and reservoirs of viruses and parasitic diseases.

New compositions with prominent acaricidal qualities, the majority of which belong to the group of synthetic pyrethroids, are being constantly created against ticks. In the search of efficient compositions to fight against ixodic ticks, the main focus was placed on acaricides that correspond to the necessary safety requirements. The measures to fight against ixodic ticks in the region were mainly caused by the fact that the development and systematization, as well as the efficiency and economic feasibility of acaricidal measures against ixodic ticks were not studied for many decades. Another reason to implement the antiparasitic measures was poor condition of the territory characterized by multiple blood protozoan diseases among animals, which lead not only to the loss of efficiency, but also to the death of production animals. The acaricidal activity of the deltamethrin-based composition was studied on laboratory strains of hungry imago ticks Dermacentor reticulatus. For this purpose, ticks (11-13 specimen per each concentration under study) were put in mill gas (polyamide grid with fine cellular structure) and placed for one minute onto various emulsion concentrations and then on a dry filter paper in a Petri dish. The results of three repeated experiments were analyzed in 12-24-48-72 hours. The laboratory results on acaricide activity of ixodic ticks in chemical water emulsions allowed their further study in field conditions. The study showed that the medium-volume spraying of a $0.005 \%$ delcid water emulsion on animals in the amount of $500 \mathrm{ml}$ on adult animal and $250 \mathrm{ml}$ on young species has high protective action against ixodic ticks. The use of the specified modes allows protecting animals from the attack of ixodic ticks within 6 days. It is found that delcid acaricidal treatment is cost efficient making 450 rubles (as of 2017 prices) per one animal throughout the entire parasitizing season of ixodic ticks.
\end{abstract}

Key words-ixodic ticks; imago; protection of animals; spraying, acaricides; delcid; deltamethrin; efficacy.

\section{I.INTRODUCTION}

Six races of ixodic ticks are found in the Russian Federation: Boophilus, Dermacentor, Haemaphysalis, Hyalomma, Ixodes and Rhipicephalus [2].

Ixodic ticks have various ecology and biology, which demonstrates their fitness to living conditions. Some tick species adapted to live in a forest-bush zone, the others - in steppe, the third - in semidesert and desert, the fourth - in mountain areas, etc. and also in indoor conditions. In each zone and even within certain pastures ticks live within strictly limited habitat having the necessary abiotic and biotic conditions for life, development and reproduction. Therefore, the distribution of ticks in pastures is not diffusive, but focal (biotopes). According to its parasitism nature, ticks are divided into pasture and earth types. Pasture ticks lay eggs in a forest floor, surface soil, root area of a vegetable cover of pastures, cracks of building walls, etc. Earth ticks lay eggs in holes of rodents and other animals, in bird nests.

According to their relations with host patrons, the pasture ticks are divided into three groups: autoecious, dixenous and trixenous (or one-host, two-host and three-host) ticks.

Autoecious: on a host body ticks evolve by attaching the hungry larva until an engorged female tick leaves the host. Onehost cycle ticks are fed by hoofed animals, and in the conditions of cultivated landscapes - mainly by cattle and horses.

Dixenous: a larva, having finished its engorgement, remains attached to the host, sheds on a nymph, which leaves the host body engorged. The nymph sheds in external environment as imago.

The parasite in the imago stage attacks animals (a second host) and falls off having sucked blood. In this case the feeder circle may be limited to one or several species of hoofed animals (often large and small cattle) and birds.

Trixenous: ticks are located on the host body only during larval, nymphal and imaginal feeding and leave it congested. Hence, in the course of their development ticks change three hosts. The molting of all phases takes place outside the host body. The host circle is extensive: larvae and nymphs are fed by small mammals, birds and reptiles, and imago - by large mammals and birds.

Following the long-term study, the experts in acarology and veterinary science proposed efficient measures to prevent the suction of ixodic ticks onto farm animals, which include the following:

1. Destruction of ixodic ticks on pets.

2. Destruction of ixodic ticks in biotopes.

All these measures are aimed to limit the number of parasites in pastures and have one common objective, i.e. to create adverse conditions for ixodic ticks [3, 4, 5, 6, 10, 14]. The prevention of ixodic ticks among animals includes general measures leading to the decrease in the number of ixodic ticks in nature, such as: planned land capability classification (brush 
eradication, burning of dead wood and dry grass in early spring before nesting of birds, plowing of pastures and sowing of grass, drying-out of wetlands or high humidity areas), widespread distribution of cultivated grassland as the most efficient method of cattle breeding. The above measures shall be carried out in tick-rich territories annually prior to or after the pasture season $[5,6,7,8,9,12]$.

For small farmsteads, the less expensive, eco-friendly and efficient method may be the manual collection of ticks from animals and extermination of already stuck ticks during inspection, hygienic procedures (cleaning, washing) and milking. In this case it is possible to prevent ixodic ticks only since a tick has already attacked and began transferring the infection. To prevent the suction of ixodic ticks it is recommended to spray and wipe production animals with acaricide emulsions and solutions. Since imago is the most harmful of all phases for large mammals, including farm animals and a person, all anti-tick measures are aimed to search various methods to destruct the adult tick on the host body and in natural habitats (Figure 1-2).

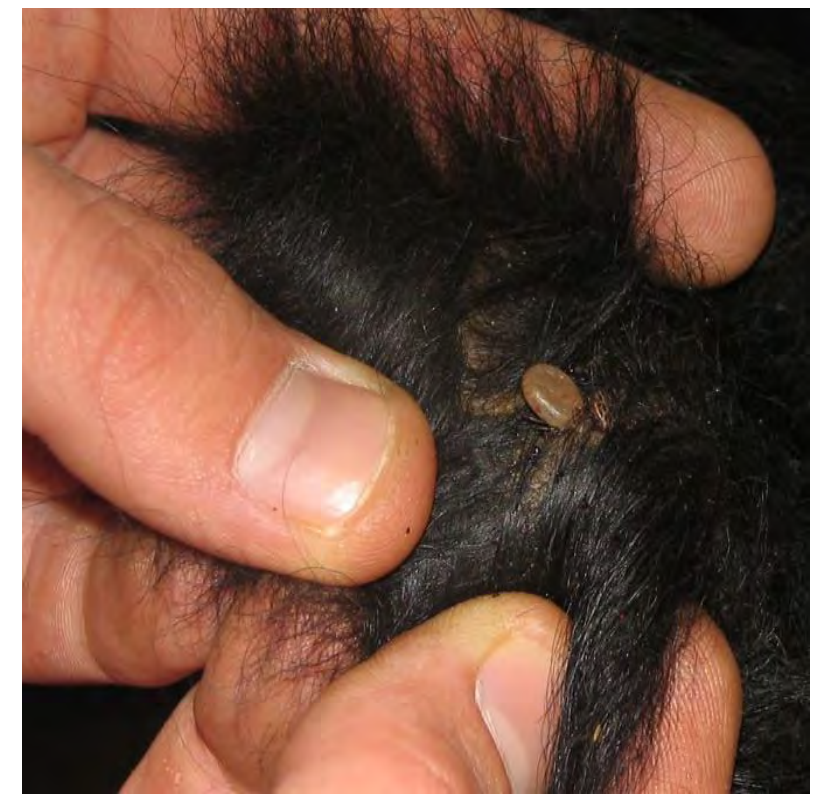

Fig. 1. Ticks nourishment on cattle auricle

To prevent the suction of ixodic ticks and transfer of infectious and invasive diseases, new compositions with prominent acaricidal qualities, the majority of which belong to the group of synthetic pyrethroids, are being constantly created $[1,4,14]$. Synthetic pyrethroids are widely used in agricultural chemistry to fight against plant pests, as well as in veterinary medicine to protect animals against blood-sucking insects [13].

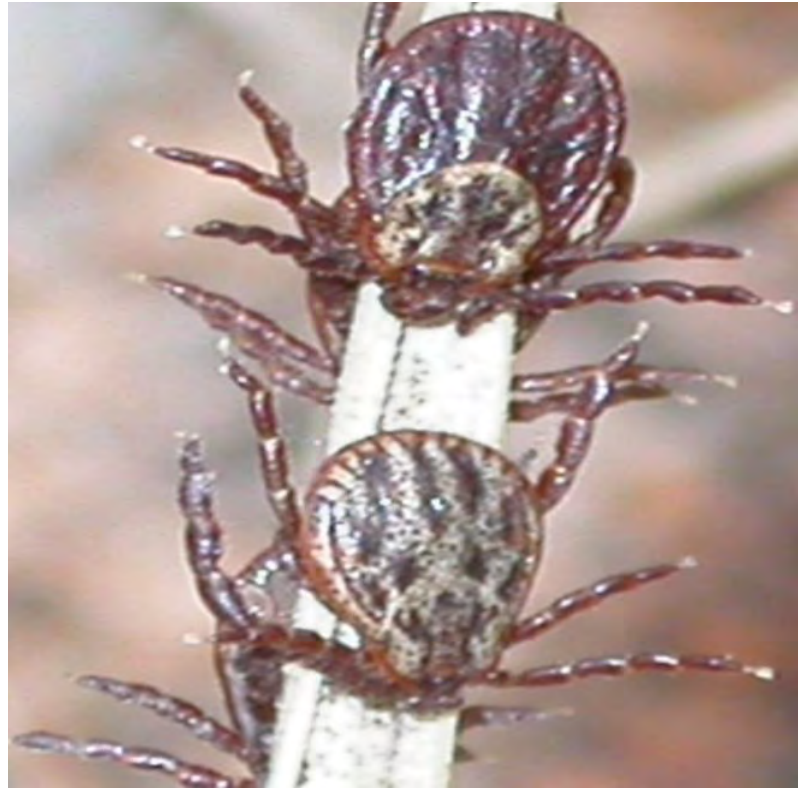

Fig. 2. Natural attack pose of a tick

During the study of efficient compositions to fight against ixodic ticks, the main attention was paid to safe acaricides suitable for agriculture. The measures to fight against ixodic ticks in the region were mainly caused by the fact that the development and systematization, as well as the efficiency and economic feasibility of acaricidal measures against ixodic ticks were not studied for many decades. Another reason to implement the antiparasitic measures was poor condition of the territory characterized by multiple blood protozoan diseases among animals triggered by ixodic ticks. The anaplasmosis or babesiosis lead to the loss of efficiency and even death of production animals $[5,6,9,15,16,17,18,19]$. All these fostered the search of means and methods to destruct ixodic ticks.

\section{II.MATERIALS AND METHODS}

The experiment was carried out from 2002 to 2015 at the Veterinary Entomology and Arachnology.

It is well-known that the imago is the most harmful of all phases of ixodic ticks. To prevent suction of ticks on animals it is advisable to use insectoacaricides by spraying or wiping the warm-blooded animals with a brush by applying special means, which are harmful to ticks - acaricides. Considering the ability of insects and ticks to be resistant to the applied insectoacaricides, there is a need to constantly change agents to ensure efficient fight with parasitic arthropods. Synthetic pyrethroids became popular among veterinary physicians and animal breeders due to their indisputable advantages: strengthening of insecticidal activity at low temperatures and small toxicity for warm-blooded animals, which is caused by fast disintegration of pyrethroids on a body of homothermal animals. All these makes pyrethroids the best agents for animal protection against ticks.

A chemical agent from the group of synthetic pyrethroids delcid was chosen and tested for the development of a system to fight against ixodic ticks. 
Delcid is an insect-acaricidal agent representing emulsible concentrate containing $4 \%$ of deltamethrin as an active substance - (S)- $\alpha$-1-cyano-3-phenoxybenzene-(1R)-cis-3-(2.2dibromophenol)-2.2-carboxylate-dimethylcyclopropane, auxiliary components and fillers. Visually it represents a uniform oily transparent yellowish-brown liquid with a slight peculiar odor, storage-resistant, forming a white stable emulsion with water, possessing enteric and contact action.

\section{RESULTS}

The acaricide activity of chemical compounds was studied on laboratory strains of hungry imago ticks Dermacentor reticulatus. For this purpose, ticks (11-13 specimen per each concentration under study) were put in mill gas (polyamide grid with fine cellular structure) and placed for one minute into various emulsion concentrations and then on a dry filter paper in a Petri dish. The results of three repeated experiments were analyzed in 12-24-48-72 hours.

It is known that the concentration of deltamethrincontaining agents is 10 times less the cypermethrin-containing ones. Therefore, five concentrations of delcid water emulsions $-0.0005 ; 0.001 ; 0.003 ; 0.005$ and $0.01 \%$ were studied in laboratory conditions. The results are shown in Table 1.

\section{TABLE 1. DELCID ACARICIDE ACTIVITY IN VITRO}

\begin{tabular}{|c|c|c|c|c|c|c|c|c|c|}
\hline \multirow[b]{3}{*}{ I } & \multirow{3}{*}{ 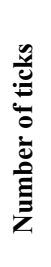 } & \multicolumn{8}{|c|}{ Acaricide activity, in one hour } \\
\hline & & \multicolumn{2}{|c|}{12} & \multicolumn{2}{|c|}{24} & \multicolumn{2}{|c|}{48} & \multicolumn{2}{|c|}{72} \\
\hline & & 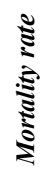 & $\%$ & 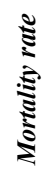 & $\%$ & 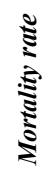 & $\%$ & 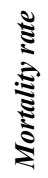 & $\%$ \\
\hline 0.0005 & 13 & 0 & 0 & 1 & 7.7 & 3 & 23.1 & 6 & 46.2 \\
\hline 0.001 & 15 & 1 & 6.7 & 2 & 13.3 & 5 & 33.3 & 13 & 86.7 \\
\hline 0.003 & 13 & 3 & 23.1 & 7 & 53.9 & 12 & 92.3 & 13 & 100 \\
\hline 0.005 & 15 & 11 & 73.3 & 15 & 100 & - & - & - & - \\
\hline 0.01 & 15 & 15 & 100 & - & - & - & - & - & - \\
\hline $\begin{array}{l}\text { Control } \\
\text { (water) }\end{array}$ & 13 & & & & clo & obs & & & \\
\hline
\end{tabular}

The laboratory tests demonstrated high efficiency of delcid water emulsions against ixodic ticks. Thus, the $0.003 \%$ delcid water emulsion led to the death of all test ticks in 72 hours, and the increase in concentration to $0.005 \%$ caused the death of all ticks in 24 hours. The concentration of active substance below $0.001 \%$ caused the death of ticks, but was ineffective. On the contrary, the increase in effective concentration twice to $0.01 \%$ was not too much different from the action in $0.005 \%$ delcid water emulsion. Water emulsions in $0.001 ; 0.003$ and $0.005 \%$ concentrations are selected for field tests.

The laboratory results on acaricide activity of ixodic ticks in delcid water emulsions allowed their further study in field conditions.

The least labor-consuming method of applying acaricides on animals is the medium-volume spraying, which has the smallest consumption of the working solution (emulsion), high manipulation speed and hence low labor costs. Besides numerous benefits of this method it has its drawbacks, such as high (in comparison with other methods) equipment cost, increased concentration and hence bigger volume of acaricide.

The working concentrations of a delcid against ixodic ticks were tested in the period of their parasitizing peak. Eighty cattle heads from among young black spotted breeds aged 8-12 months were chosen for test at various delcid concentrations. The animals participating in the experiment were split into three test and one control group 20 heads each. Prior to applying the acaricide water emulsion on animals the abundance of ixodic ticks was considered, and the groups were formed following the analogue principle: the abundance index of ticks in the first group made $25.2 \pm 2.25$ specimen, in the second $-24.1 \pm 2.08$ and in the third $-29.2 \pm 2.42$ specimen per one animal. In the control group the abundance index made $19.3 \pm 1.64$ specimen. The first group was treated with $0.001 \%$ delcid water emulsion (for active substance), the second $-0.003 \%$ delcid water emulsion (for active substance), and the third $-0.005 \%$ delcid water emulsion (for active substance). The agent was applied once only via medium-volume spraying from Oleo-mac device in the amount of $500 \mathrm{ml}$ per one animal. The control animals were treated with water in volumes similar to test animals (Table 2)

. TABLE 2. DELCID ACARICIDE ACTIVITY UNDER PRODUCTION CONDITIONS

\begin{tabular}{|c|c|c|c|c|c|c|c|}
\hline \multirow{2}{*}{ 氖 } & \multirow{2}{*}{$\begin{array}{l}\text { Abundance index } \\
\text { per one animal } \\
(n=20)\end{array}$} & \multicolumn{3}{|c|}{ Number of ticks after treatment, in one hour } & \multicolumn{3}{|c|}{ Intensive efficacy, $\%$, in one hour } \\
\hline & & 24 & 48 & 72 & 24 & 48 & 72 \\
\hline 0.001 & $\begin{array}{c}25.2 \\
\pm 2.25 \\
\end{array}$ & $\begin{array}{c}23.2 \\
\pm 2.25 \\
\end{array}$ & $\begin{array}{c}21.9 \\
\pm 2.20 \\
\end{array}$ & $\begin{array}{c}8.2 \\
\pm 0.84 \\
\end{array}$ & $\begin{array}{c}7.9 \\
\pm 2.05 \\
\end{array}$ & $\begin{array}{c}13.1 \\
\pm 2.82 \\
\end{array}$ & $\begin{array}{r}67.5 \\
\pm 1.12 \\
\end{array}$ \\
\hline 0.003 & $\begin{array}{c}24.1 \\
\pm 2.08 \\
\end{array}$ & $\begin{array}{c}18.2 \\
\pm 1.85 \\
\end{array}$ & $\begin{array}{c}11.9 \\
\pm 1.20 \\
\end{array}$ & $\begin{array}{c}2.1 \\
\pm 0.30 \\
\end{array}$ & $\begin{array}{c}24.5 \\
\pm 2.07 \\
\end{array}$ & $\begin{array}{c}50.6 \\
\pm 1.23 \\
\end{array}$ & $\begin{array}{r}91.3 \\
\pm 0.38 \\
\end{array}$ \\
\hline 0.005 & $\begin{array}{c}29.2 \\
\pm 2.42\end{array}$ & $\begin{array}{c}16.0 \\
\pm 1.57\end{array}$ & $\begin{array}{c}2.2 \\
\pm 0,44\end{array}$ & 0 & $\begin{array}{c}45.2 \\
\pm 1.88\end{array}$ & $\begin{array}{c}92.5 \\
\pm 0.48\end{array}$ & 100 \\
\hline water & $\begin{array}{c}19,3 \\
\pm 1.64\end{array}$ & $\begin{array}{c}19.3 \\
\pm 1.64\end{array}$ & $\begin{array}{c}19.3 \\
\pm 1,64\end{array}$ & $\begin{array}{c}19.8 \\
\pm 1.82\end{array}$ & - & - & - \\
\hline
\end{tabular}


All animals were observed throughout the entire experiment. The intoxication symptoms were noted during the entire period of observation (72 hours) after applying the agent.

In 24 hours after applying delcid water emulsions on animals of the first test group the quantity of ixodic ticks decreased by $7.9 \pm 2.05 \%$, the abundance index made $23.2 \pm 2.25$ specimen. The quantity of ixodic ticks for animals of the second group was reduced by $24.5 \pm 2.07 \%$, and the abundance index made $18.2 \pm 1.85$ specimen. For the third group the quantity of parasites was reduced almost by half (intensive efficacy $45.6 \pm 1.88 \%$ ), and the abundance index made $16.0 \pm 1.57$ specimen.

In 48 hours after treatment the abundance index of animals of the first test group was reduced to $21.9 \pm 2.20$ specimen (intensive efficacy $13.1 \pm 2.82 \%$ ). The abundance of parasites was reduced for animals of the second test group and made $11.9 \pm 1.20$ specimen (intensive efficacy $50.6 \pm 1.23 \%$ ). The abundance index for animals of the third test group on the second day made $2.2 \pm 0.44$ specimen (intensive efficacy $92.5 \pm 0.48 \%)$.

In 72 hours after applying delcid water emulsions it was found that the abundance index of ixodic ticks for animals of the first test group made $8.2 \pm 0.84$ specimen (intensive efficacy $67.5 \pm 1.12 \%$ ). For the second group of animals the abundance index of ixodic ticks made $2.1 \pm 0.30$ specimen (intensive efficacy $91.3 \pm 0.38 \%$ ). For the third test group ticks were not revealed (intensive efficacy $-100 \%$ ).

The abundance index of ixodic ticks for animals in the control group at the beginning of the experiment made $19.3 \pm 1.64$ specimen. Throughout the entire period of observation, the quantity of ticks was slightly increasing, which was caused by the attack of hungry specimen.

The field study of acaricide efficacy of delcid made it possible to conclude that its $0.005 \%$ water emulsion is highly efficient against ixodic ticks when the animals are treated via $500 \mathrm{ml}$ medium-volume spraying.

Considering the average duration of parasitizing of ixodic ticks in the Northern Trans-Ural region (116 \pm 5.36 days during the season), it was possible to calculated the cost of acaricide treatment for cattle. It was found that delcid acaricidal treatment is cost-efficient making 450 rubles (as of 2017 prices) per one animal throughout the entire parasitizing season of ixodic ticks

\section{CONCLUSIONS}

Laboratory tests allowed selecting three effective delcid concentrations: $0.001 ; 0.003$ and $0.005 \%$, not all being efficient during field tests. Thus, during field tests a high protective effect against ixodic ticks was recorded at the medium-volume treatment with $0.005 \%$ delcid water emulsion. The study of residual acaricide action made it possible to define the frequency of applying working solutions on animals thus making 6 days. The cost of acaricide treatment with $0.005 \%$ delcid water emulsion per one animal made 450 rubles.

\section{Acknowledgements}

The paper was prepared under financial support of the FAO of Russia within the framework of the topics of FNI No. 0371-
2018-0037 and the Basic Research Program of the Russian Academy of Sciences, registration number AAAA-A18118020690239-7 "Study of the effectiveness of new antiparasitic preparations".

\section{References}

[1] V.M. Arisov, K.G. Kurochkina, M.V. Kharisov, K.G. Kurochkin "Immunological and allergenic properties delcid", Veterinary Medicine, vol. 3, pp. 33-37, 2007.

[2] Yu. S. Balashov, "Blood-sucking ticks (Ixodidae)", vectors of diseases of humans and animals. L.,.pp. 319, 1967.

[3] P. Biglari, H. Bakhshi, S. Chinikar, H. Belqeiszadeh, M. Ghaffari, S. Javaherizadeh, F. Faghihi, Z. Telmadarraiy, Hyalomma anatolicum as the Main Infesting Tick in an Important Livestock Rearing Region, Central Area of Iran. Iran J Public Health, vol. 47(5): pp. 742-749, 2018

[4] P. Bourdeau, T. Ximenes, O. Beziade., "Characteristics of Dermacentor reticulatus and Ixodes ricinus infestations in 191 dogs in France", Veterinary Dermatology, vol. 15, issues 1 on pp. 49-49, Aug. 2004.

[5] Yu.V. Glazunov1, L.A. Glazunova, "Phenology of Pasture Ticks in The Trans-Urals”, Indian Vet. J., 95 (01), pp. 19-22, 2018.

[6] L.A. Glazunova, Yu.V Glazunov, A.A. Ergashev, "Ecologicalepizootical situation on telasiosis among large cattle in Northern Ural region", Research Journal of Pharmaceutical, Biological and Chemical Sciences. T. 9. Vol. 4. pp. 1687-1693, 2018.

[7] G.G. Grigor, A.A. Zemtsov, Natural "zoning of Western Siberia" questions of geography, Publishing house of the USSR Academy of Sciences, 1961. - pp. 55

[8] M.A. Chasovshchikova, O.M. Sheveleva, M.A. Svjazhenina, N.I. Tatarkina, A.B. Satkeeva, A.A. Bakharev, E.A. Ponomareva, A.G. Koshchaev, "Relationship between the genetic variants of kappacasein and prolactin and the productive-biological characteristics of cows of the black-motley breed" Journal of Pharmaceutical Sciences and Research. T. 9. Vol. 7. pp. 1038-1044, 2017

[9] L.N. Karetin, Soil Tyumen region, Novosibirsk.: Science., 1990, pp 281 .

[10] P.I. Khristinovsky, V.V. Belimenko, P.I. Khristinovsky, V. Bulimenko, "Piroplasmosis animals in the southern Urals", Russian parasitological magazine. vol. 2. - pp. 70-74, 2009.

[11] N.E. Kosminkov, B.K. Laipanov, V.N. Domacki, V. Belimenko, Parasitology and parasitic diseases of farm animals Textbook, Moscow, 2017.

[12] E.T. Machtinger, A.Y. Li, Evaluation of four commercial natural products for repellency and toxicity against the lone star tick, Amblyomma americanum (Acari: Ixodidae), vol. 73(3-4), pp. 451460, doi: 10.1007/s10493-017-0185-z. Epub, vol. 22, 2017.

[13] L.P. Maestas, S.E Mays, H.B. Britten, L.D. Auckland, S.A. Hamer, Surveillance for Ixodes scapularis (Acari Ixodidae) and Borrelia burgdorferi in Eastern South Dakota State Parks and Nature Areas.J Med Entomol, 25. doi: 10.1093/jme/tjy101, 2018.

[14] O.A. Phedorova, T.A. Khlyzova, "Efficiency of systematic spraying of cattle with decidium against bloodsucking midges on pastures", Vestnik Krasnoyarsk State Agrarian University. vol. 2 (113), pp. 159164,2016

[15] R.I. Rodriguez-Vivas, N.N. Jonsson, C. Bhushan, "Strategies for the control of Rhipicephalus microplus ticks in a world of conventional acaricide and macrocyclic lactone resistance", Parasitol Res,vol. 117(1), 2018, vol. 20, 2017.

[16] L. Skipin, E. Gaevaya, E. Zaharova, V. Petukhova, K. Sidorova "Biogeochemistry of heavy metals in trophic chain in terms of the south of Tumen region" // Procedia Engineering (see in books). Vol. 165. pp. 860-868, 2016.

[17] O.A. Stolbova, Yu.V. Glazunov, L.N. Skosyrskikh, "Ticks-parasites of dogs in Northern Trans-Urals", Indo American journal of pharmaceutical sciences. vol. 05 (03), pp.1675-1682, 2018.

[18] Z. Széll, Z. Sréter-Lancz, K. Márialigeti, T. Sréter, "Temporal distribution of Ixodes ricinus", Dermacentor reticulatus and 
Haemaphysalis concinna in Hungary, Veterinary Parasitology, vol. 141 , on pp. 377-379, 2006.
[19] M.Yu. Tohou, S.N. Lutsuk, Yu.V. Dyachenko, Yu. M. Torkhov, S. N. Lutsuk, V. Dyachenko, "Phenology of ticks of the genus Dermacentor in the Central Ciscaucasia", Parasitology, Vol. 47, 6, pp. 437-447, 2013. 